This item was submitted to Loughborough's Research Repository by the author.

Items in Figshare are protected by copyright, with all rights reserved, unless otherwise indicated.

\title{
Parametric amplification of vortex-antivortex pair generation in a Josephson junction
}

PLEASE CITE THE PUBLISHED VERSION

http://dx.doi.org/10.1103/PhysRevB.90.134505

\section{PUBLISHER}

(C) The American Physical Society

\section{VERSION}

VoR (Version of Record)

\section{PUBLISHER STATEMENT}

This work is made available according to the conditions of the Creative Commons Attribution-NonCommercialNoDerivatives 4.0 International (CC BY-NC-ND 4.0) licence. Full details of this licence are available at: https://creativecommons.org/licenses/by-nc-nd/4.0/

\section{LICENCE}

CC BY-NC-ND 4.0

\section{REPOSITORY RECORD}

Berdiyorov, G.R., M.V. Milosevic, Sergey Savel'ev, F.V. Kusmartsev, and F.M. Peeters. 2019. "Parametric Amplification of Vortex-antivortex Pair Generation in a Josephson Junction". figshare. https://hdl.handle.net/2134/18511. 


\title{
Parametric amplification of vortex-antivortex pair generation in a Josephson junction
}

\author{
G. R. Berdiyorov, ${ }^{1,2}$ M. V. Milošević, ${ }^{3}$ S. Savel'ev, ${ }^{1}$ F. Kusmartsev, ${ }^{1}$ and F. M. Peeters ${ }^{3, *}$ \\ ${ }^{1}$ Department of Physics, Loughborough University, Leicestershire, LE11 3TU, United Kingdom \\ ${ }^{2}$ Qatar Environment and Energy Research Institute, Doha, Qatar \\ ${ }^{3}$ Departement Fysica, Universiteit Antwerpen, Groenenborgerlaan 171, B-2020 Antwerpen, Belgium \\ (Received 2 July 2013; revised manuscript received 23 December 2013; published 6 October 2014)
}

\begin{abstract}
Using advanced three-dimensional simulations, we show that an Abrikosov vortex, trapped inside a cavity perpendicular to an artificial Josephson junction, can serve as a very efficient source for generation of Josephson vortex-antivortex pairs in the presence of the applied electric current. In such a case, the nucleation rate of the pairs can be tuned in a broad range by an out-of-plane ac magnetic field in a broad range of frequencies. This parametrically amplified vortex-antivortex nucleation can be considered as a macroscopic analog of the dynamic Casimir effect, where fluxon pairs mimic the photons and the ac magnetic field plays the role of the oscillating mirrors. The emerging vortex pairs in our system can be detected by the pronounced features in the measured voltage characteristics, or through the emitted electromagnetic radiation, and exhibit resonant dynamics with respect to the frequency of the applied magnetic field. Reported tunability of the Josephson oscillations can be useful for developing high-frequency emission devices.
\end{abstract}

DOI: 10.1103/PhysRevB.90.134505

PACS number(s): 74.81.Fa, 73.23.-b, 74.25.Uv, 74.78.Na

\section{INTRODUCTION}

The fundamental process of parametric amplification suitably exploits periodic variation of a parameter to achieve gain in certain modes of a given system and is relevant to many physical phenomena [1]. It has also found various applications in electronics, where the demand for low-noise solutions has introduced superconducting circuitry [2] — achieving parametric amplification utilizing the current-dependent kinetic inductance (see, e.g., Ref. [3]) or response to the applied magnetic field [4].

Already since the late 1960s, parametric amplification has found one of its main applications, in nonlinear optics [5], and still attracts considerable interest [6,7]. About the same time, the role of the periodic excitations required to trigger otherwise unachievable phenomena in optics was examined in what is now known as the dynamic Casimir effect (DCE) [8], where the virtual photons in vacuum, associated with quantum fluctuations, are converted under interaction with the fast oscillating mirrors into experimentally detectable real photons. Subsequently, the DCE concept of generation of quasiparticles has been explored in a plethora of systems. In optical systems, proposals were made to suitably vary the boundary conditions rather than vibrate the optical cavity (see, e.g., Refs. [9-14]). The DCE was recently experimentally realized in a superconducting circuit containing a coplanar transmission line, where the role of mirrors for the usual DCE was played by the modulating inductance of a superconducting quantum interference device [15]. Fast variations of boundary conditions mimicking accelerating mirrors were also used in an acoustic analog of the DCE in Bose-Einstein condensates (BECs) by changing the scattering length of the interacting Bose gas [16,17]. Several other analogs of the DCE were reported, in different systems where the stringent requirements for its experimental demonstration are eased [18-21]. Particu-

\footnotetext{
*francois.peeters@untwerpen.be
}

larly superconductors [18] and BECs [22-24] are attractive in this respect, because of the pronounced macroscopic observables of quantum effects at low temperatures.

In this article we study the parametric amplification of the generation of Josephson vortex-antivortex pairs in layered superconductors by an external ac magnetic field and in the presence of applied current, and we explore the applicability of the DCE principles on the control and tunability of this effect. Since, to some extent, one could consider the DCE as a quantum counterpart of the classical parametric amplification, our system exhibits a macroscopic and first classical analog of the DCE, in contrast to other proposed analogs [18-24] where quantum fluctuations do play an important role. Vortexantivortex pairs have been intensively studied in superconductors with magnetic nanostructures on top, where they appear and stabilize under the influence of the inhomogeneous stray magnetic field [25], have been directly imaged in experiment [26], and exhibit peculiar dynamics under the applied electric current [27]. Actually, vortex-antivortex pairs can be induced by electric current even in the absence of the magnetic field, in effect which is hydrodynamically similar to vortex shedding in superfluids [28], where the rate of nucleated pairs and their subsequent annihilation depends on the magnitude of the applied current [29]. A similar principle has been recently used to generate Josephson vortex-antivortex pairs in a planar junction [30], where another degree of tunability was demonstrated by using a preconditioned phase imprint on the junction. Adding the fact that vortex-antivortex pairs are known to appear in Josephson junctions also under thermal and quantum fluctuations [31], and that the dynamics of such vortices under external excitations is intimately related to the properties and coherence of the emitted electromagnetic radiation from layered materials [32], it is of timely interest to see to which extent the nucleation and motion of Josephson vortex-antivortex pairs can be controlled by experimentally accessible tools.

Therefore, in this article we consider the creation of Josephson vortex-antivortex (V-Av) pairs in a planar Josephson 


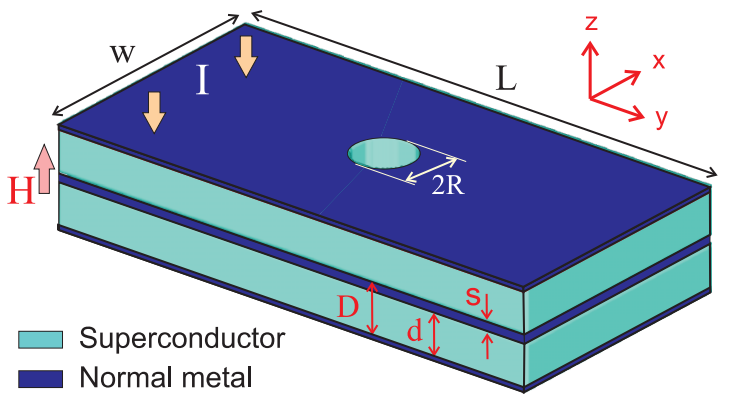

FIG. 1. (Color online) Oblique view of the system: two superconducting layers (of thickness $d \ll \lambda$ ) with a normal metal junction (of thickness $s \ll \xi, \lambda$ ) in between, and external current $I$ and magnetic field $H$ applied perpendicularly to the junction plane. The shown hole of radius $R$ is designed to trap Abrikosov vortices.

junction under applied electric current and (ac) magnetic field, and we achieve the control of the nucleation rate by tuning the frequency of the applied magnetic field. Our system is an artificial Josephson junction between two thin superconducting layers, with an Abrikosov vortex trapped inside a hole perforating the entire structure (see Fig. 1). As already mentioned, we explore here the applicability of the DCE principles on the generation of the pairs. The externally applied current excites the "vacuum state" of the system close to the pair nucleation point (see, e.g., Ref. [20]). Note however that fluctuations are not needed for the pair nucleation in our system; thus, the here discussed macroscopic classical analog of the DCE can be always regarded as simply a vortex-antivortex parametric amplifier. As we show below, the applied out-of-plane magnetic field switches this "vacuum" into a new state, containing fluxons which appear as pairwise excitations. The Abrikosov vortex plays an important role in this process, determining the position of the pair nucleation. A sinusoidally modulated out-of-plane magnetic field provides the time-dependent boundary condition, creating an effect which is similar to the moving mirrors in the original DCE proposal $[33,34]$. This boundary condition is enforced in practice by the screening Meissner currents, which affect the phase distribution around the pinned Abrikosov vortex and thereby affect the generation of fluxon pairs. Our system is very convenient to further study V-Av nucleation and dynamics in experiment, being easy to fabricate, where generation of the pairs can be monitored via temporal features in the measured voltage and where emitted radiation from the generated pairs [35] can be detected either directly [36,37] or via resonant features in the current-voltage characteristics of the system [38,39].

The article is organized as follows. In Sec. II, we outline the theoretical formalism and practical issues concerning the simulations. Section III is devoted to the characterization of the system in the presence of dc electric current and an out-of-plane magnetic field, both its stationary and resistive state. In Sec. IV, we present the achieved tunability and control of the generated V-Av pairs in the Josephson junction under the ac magnetic field and the resonance effects between the nucleation frequency and the frequency of the drive. Our results are summarized in $\mathrm{Sec}$. V.

\section{THEORETICAL APPROACH}

To simulate the behavior of our system, we solved the timedependent Ginzburg-Landau (GL) equation:

$$
u(\partial / \partial t+i \varphi) \psi=(\nabla-i \mathbf{A})^{2} \psi+\left(1-|\psi|^{2}\right) \psi,
$$

where $\psi$ is the superconducting order parameter, $\varphi$ the electrostatic potential, and $\mathbf{A}$ the magnetic vector potential, with the following boundary condition for the Josephson tunneling [40],

$$
\begin{aligned}
& \left.\left(-i \nabla_{z}-A_{z}\right) \psi\right|_{\perp} ^{\text {bot }} \\
& =\frac{i}{\mu s}[\psi(x, y, D) \exp (-i \bar{A} s)-\psi(x, y, d)] \\
& \left.\left(-i \nabla_{z}-A_{z}\right) \psi\right|_{\perp} ^{\text {top }} \\
& =\frac{i}{\mu s}[\psi(x, y, d) \exp (i \bar{A} s)-\psi(x, y, D)],
\end{aligned}
$$

at the interface of the junction area with the bottom and top superconducting layers (each layer of thickness $d$, separated by a metallic junction of thickness $s$, see Fig. 1), respectively. Here $D=d+s, \bar{A}$ is defined as $\bar{A} \equiv(1 / s) \int_{d}^{D} A_{z} d z$, and $\mu$ is the ratio of the mass of the Cooper pairs in the metallic and superconducting regions. The superconducting-vacuum boundary condition $\left.\vec{n}(-i \nabla-\mathbf{A}) \psi\right|_{n}=0$ is applied at the other boundaries with $\vec{n}$ being the unit vector normal to the surface. Equation (1) is coupled with the equation for the electrostatic potential $\varphi$ :

$$
\Delta \varphi=\operatorname{div}\left(\mathbf{j}_{\mathbf{s}}\right)
$$

where the superconducting current is given by

$$
\mathbf{j}_{\mathbf{s}}=\left[\left(\psi^{*} \nabla \psi-\psi \nabla \psi^{*}\right)-\mathbf{A}|\psi|^{2}\right] / 2 i,
$$

with a Josephson component $j_{s \perp}$ :

$$
\begin{aligned}
j_{s \perp}= & {\left[\psi(x, y, D) \exp (-i \bar{A} s) \psi^{*}(x, y, d)\right.} \\
& \left.-\psi^{*}(x, y, D) \exp (i \bar{A} s) \psi(x, y, d)\right] / 2 i \mu s .
\end{aligned}
$$

The external current is injected through the metallic leads, as shown in Fig. 1, using the boundary conditions of $\psi=0$ and $\left.\nabla \varphi\right|_{n}= \pm j$, with $j$ being the uniformly applied current density. In all equations, the length is expressed in units of the superconducting coherence length $\xi$ and the vector potential is scaled to $\Phi_{0} /(2 \pi \xi)$, where $\Phi_{0}$ is the magnetic flux quantum. Time is scaled to units of $t_{\mathrm{GL}}=4 \pi \lambda^{2} / c^{2} \rho_{n}$ ( $\rho_{n}$ is the normal-state resistivity and $\lambda$ is the magnetic field penetration depth), the electrostatic potential to $V_{0}=c \Phi_{0} \rho_{n} / 8 \pi^{2} \lambda \xi$, and the current density is measured in $j_{0}=c \Phi_{0} / 8 \pi^{2} \lambda^{2} \xi$. The parameters $u$ and $\mu$ are taken as $u=5.79$ [41] and $\mu=1$. Using $\xi(0)=10 \mathrm{~nm}, \lambda(0)=200 \mathrm{~nm}$, and $\rho_{n}=18.7 \mu \Omega \mathrm{cm}$, which are typical for $\mathrm{Nb}$ thin films [42], we obtain $t_{0} \approx$ 2.69 ps and $V_{0}=0.12 \mathrm{mV}$ close to the critical temperature. We neglected demagnetization effects, which is valid for extreme type-II superconductors. The coupled Eqs. (1)-(3) are solved self-consistently following the numerical procedure of Ref. [40].

Note that we have neglected heating effects in our analysis assuming that the sample is in good contact with the substrate. Thermal fluctuations can also be disregarded in conventional low- $T_{c}$ superconductors (see, e.g., Ref. [43]), thus we do not 
expect thermally activated V-Av pairs in artificial Josephson junctions made of low- $T_{c}$ materials at temperatures below $0.8 T_{c}$ (for high- $T_{c}$ superconductors, this threshold temperature is lowered, likely to below $0.5 T_{c}$ ). We also expect no qualitative changes in our findings if the lateral dimensions of the sample are smaller than the thermal length.

\section{BEHAVIOR UNDER APPLIED dc CURRENT AND MAGNETIC FIELD}

As a typical example we consider a sample with lateral dimensions $L=100 \xi, w=20 \xi, d=5 \xi$, and $s=0.4 \xi$ and a hole of size $R=1.6 \xi$. In such a setup, the Abrikosov vortex can be inserted using different experimental techniques (e.g., by field cooling, by passing a large current through the system [44], or by using laser or electron beams [45]) and is trapped by the hole. It is already well known that a small perpendicular magnetic field can strongly affect both the static and the dynamic properties of the Josephson junction, due to the penetration of vortices (also known as pancake vortices in the case of very thin layers) [44-53]. Their effect is strongly reduced by introducing columnar defects [47], which align the Abrikosov vortices perpendicularly to the junction, thus diminishing the phase and magnetic field variations across the junction. Expecting no phase difference across the junction, we first study the effect of the vortex trapped inside the hole on the critical parameters of the system and show the obtained current-voltage $(I-V)$ characteristics in Fig. 2. We observe a noticeable decrease of the critical current of the system (labeled $j_{c}$, indicating current for which resistance arises) when the Abrikosov vortex is present (compare black/solid and red/open dots in Fig. 2), despite the fact that no misalignment of the vortex is allowed due to the pinning and no phase difference is imposed on the junction by this straight vortex. In addition, the critical current shows a strong dependence on the polarity of the applied out-of-plane magnetic field (see

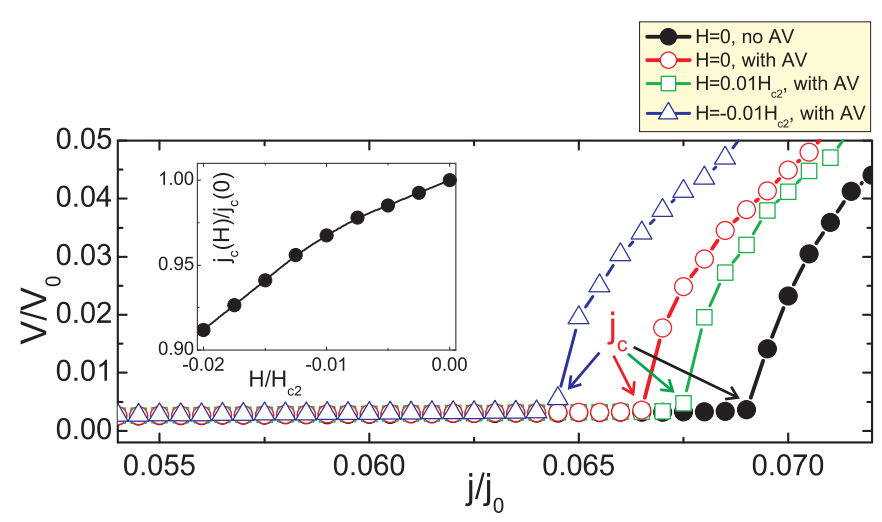

FIG. 2. (Color online) Time-averaged voltage vs applied current density $(I-V)$ characteristics of the sample without (solid circles) and with (open symbols) an Abrikosov vortex trapped in the hole of radius $R=1.6 \xi$. The length of the sample is $L=100 \xi$, the thickness of the superconducting layers is $d=5 \xi$, and the thickness of the normal layer is $s=0.4 \xi$. The out-of-plain magnetic field $H$ is applied either in the positive (open squares) or the negative (open triangles) direction. The inset shows the field dependence of the critical current $j_{c}$.
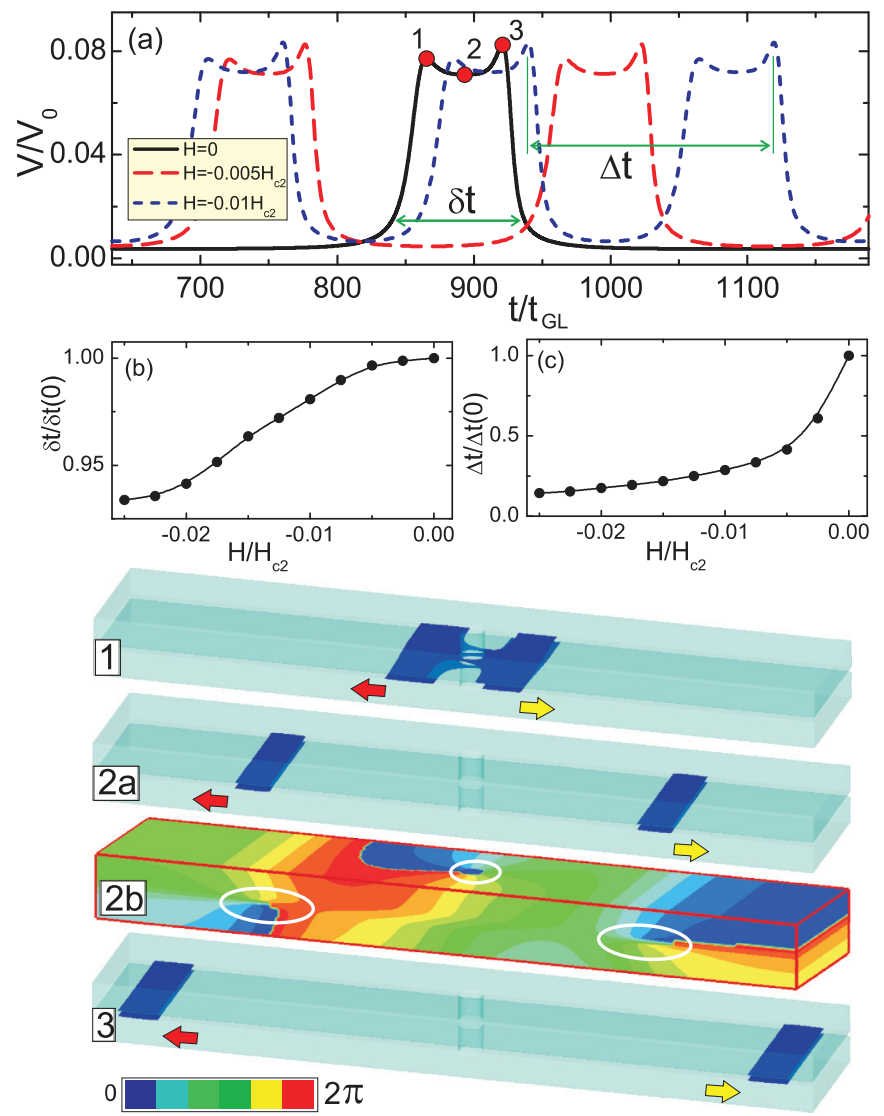

FIG. 3. (Color online) (a) Voltage vs time characteristics $[V(t)]$ of the sample with dimensions $L=100 \xi, w=20 \xi, d=5 \xi, s=$ $0.4 \xi$, and $R=1.6 \xi$, in the presence of a trapped Abrikosov vortex, for $j>j_{c}$ (fixed at $j=0.067 j_{0}$ ), and three values of the magnetic field $H$. Voltage is measured in $V_{0}=c \Phi_{0} \rho_{n} / 8 \pi^{2} \lambda \xi$, current density in $j_{0}=c \Phi_{0} / 8 \pi^{2} \lambda^{2} \xi$, and time in $t_{\mathrm{GL}}=4 \pi \lambda^{2} / c^{2} \rho_{n}$ ( $\rho_{n}$ is the normalstate resistivity). (b) Characteristic crossing time of a Josephson fluxon over half the length of the sample, $\delta t$, as a function of $H$. (c) Period of the voltage oscillations $\Delta t$ as a function of $H$. Panels 1 , $2 \mathrm{a}$, and 3 show isosurface plots of the Cooper-pair density (isovalue is $10 \%$ of $\left.|\psi|_{\max }^{2}\right)$ for $H=0$ at times indicated on the $V(t)$ curve. Panel $2 \mathrm{~b}$ shows the contour plot of the phase of the order parameter at the surface of the sample at time indicated by point 2 . White circles indicate the position of the vortices and arrows indicate the direction of motion of Josephson V-Av pairs.

data plotted with green squares and blue triangles in Fig. 2), which is not found in the absence of the trapped vortex. Namely, $j_{c}$ increases for positive direction of the magnetic field, when the screening Meissner currents compensate the circulating currents of the trapped vortex. Strong reduction of $j_{c}$ is observed for negative polarity of the field (see also inset of Fig. 3) where Meissner currents superpose on the supercurrent of the vortex. Note that the presence of the empty hole or the local suppression of superconductivity near the hole cannot explain these findings, because in either case $j_{c}$ should not depend on the field polarity. Moreover, similar columnar defects are known to have a negligible effect on the properties of the stacked Josephson junctions [47].

Similar effects of Abrikosov vortices on the critical current in layered superconductors have been investigated in the 
past [44-53]. In the present study, we focus on the nucleation and evolution of the resistive regime (i.e., at applied currents in the vicinity and above $j_{c}$ ). Figure 3(a) shows the time evolution of the output voltage for an applied current just above $j_{c}$ and no applied magnetic field, together with isosurface plots of the Cooper-pair density which reveal that dissipation arises from the periodic nucleation, motion, and annihilation of $\mathrm{V}-\mathrm{Av}$ pairs (see panels 1-3). The pairs nucleate in the middle of the sample, where the Abrikosov vortex is pinned (see panel 1), resulting in a peak in the measured voltage (point 1). Fluxons are driven apart towards the sample edges by the applied current (panel 2a), where they leave the sample (panel 3 ) with a corresponding local maximum in the $V(t)$ curve (point 3). Panel 2b shows contour plots of the phase of the order parameter at the outer surfaces of the sample, where the positions of the moving Josephson vortices and the pinned Abrikosov vortex are indicated by white circles. Note that such vortex-antivortex mechanism is very similar to a vortex shedding effect under external driving in superfluids (e.g., in BECs, see Ref. [54]). Note also that in the absence of the Abrikosov vortex the Josephson fluxons nucleate at the edges, travel through the junction, and annihilate in the middle of the sample $[30,40]$.

Next we investigate the effect of the applied out-of-plane magnetic field $H$ on the dynamics of the Josephson V-Av pairs. The voltage-time characteristics of the system for two nonzero values of $H$ are plotted in Fig. 3(a) as red/dashed and blue/dotted curves. As in the absence of field (solid black curve), $V(t)$ shows periodic oscillations with two peaks in each period, corresponding to periodic nucleation and expulsion of $\mathrm{V}-\mathrm{Av}$ pairs. Surprisingly, the characteristic time of the system $\delta t$, determined by the "lifetime" of the pairs (i.e., time for a vortex/antivortex to leave the sample upon nucleation), depends very weakly on the magnetic field strength. We find a less than $7 \%$ decrease of $\delta t$ for the considered range of magnetic field [see Fig. 3(b)]. However, the period of voltage oscillations decreases dramatically with increasing magnetic field, as shown in Fig. 3(c), indicating that the nucleation rate of $\mathrm{V}$-Av pairs sharply increases. For the considered value of the current and the applied magnetic field varied in the range $H / H_{c 2}=0.0-0.025$, the frequency of the voltage oscillations was in the range $v=1.24 \times 10^{-3}-8.73 \times 10^{-3} t_{\mathrm{GL}}^{-1}$. For a sample made of thin $\mathrm{Nb}$ films [42] the frequency of the oscillations was estimated to be in the $v=0.45-3.25 \mathrm{GHz}$ range, i.e., easily tunable by an order of magnitude.

\section{TUNABILITY AND RESONANCE IN ac MAGNETIC FIELD}

In this section, we show that nucleation of Josephson V-Av pairs in our system can indeed mimic the photon generation in optical systems, in the sense that the nucleation can be induced and controlled by oscillating boundary conditions, in analogy to the DCE effect due to moving mirrors. We apply an oscillating magnetic field $H=-H_{\mathrm{dc}}+H_{\mathrm{ac}} \sin (2 \pi \nu t)$, where both dc and ac components of the field are much smaller than the penetration field of Abrikosov vortices in the system, and consider smaller applied current then the critical one for that applied field $\left(j<j_{c}\right)$. In other words, V-Av pairs will not be generated, neither by field $H_{\mathrm{dc}}$ nor current $j$, nor
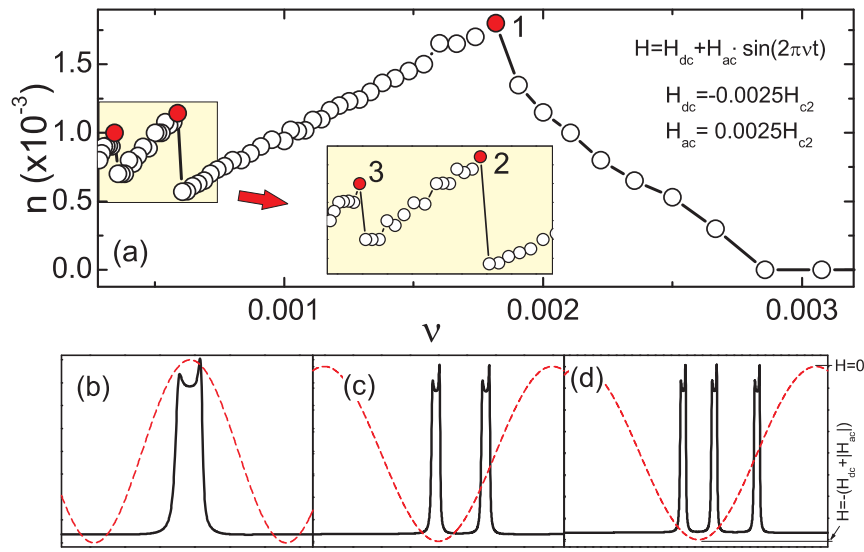

FIG. 4. (Color online) (a) Number of Josephson V-Av pairs generated per unit time, $n$, as a function of the frequency of the oscillating magnetic field $H=H_{\mathrm{dc}}+H_{\mathrm{ac}} \sin (2 \pi v t)$ for $H_{\mathrm{dc}}=-0.0025 H_{c 2}$. The current $j=0.066 j_{0}=0.99 j_{c}$ and the ac field component is $H_{\mathrm{ac}}=0.0025 H_{c 2}$. The sample parameters are the same as those in Fig. 3. The insets show the enlargement of the lower frequency part of the $n(v)$ curve. (b-d) $V(t)$ curves of the sample for the frequencies of the magnetic field indicated in panel (a) by points $1-3$, respectively. Dashed curves show the profile of the ac magnetic field in arbitrary units.

their combination-instead, they will appear only under the influence of the ac field. To characterize the nucleation process of the V-Av pairs, we plotted in Fig. 4(a) the pair-creation rate, i.e., the number of pairs detected at the edges of the sample per unit time, $n$, as a function of the frequency $v$ of the ac field. We observe a clear resonance maximum in the $n(v)$ curve (see point 1) when one $\mathrm{V}$-Av pair nucleates per single period of the field oscillations [see Fig. 4(b)]. This is one of the classical parametric resonance conditions (see, e.g., Ref. [55]) of vortex-antivortex generation, which can be viewed as related to the DCE - where, in contrast to the discussed here classical DCE analog, the vacuum fluctuations of the electromagnetic field in an optical cavity are exponentially amplified. The value of $n$ decreases with further increasing $v$, and no pairs are nucleated at larger frequencies, as also predicted for the other model systems for the DCE (see, e.g., Ref. [18]). The number $n$ also decreases with decreasing $v$ away from the resonance peak. However, at very low frequencies we observe additional peaks in the $n(v)$ curve [see inset of Fig. 4(a)]. Analysis of the voltage curves shows that [see Figs. 4(c) and 4(d)] for these frequencies the number of $\mathrm{V}-\mathrm{Av}$ pairs nucleated per single period of field increases by one. Note that in this low-frequency range the system responds only to the largest value of the negative field (i.e., adiabatic response), whereas in the parametric resonance case out-of-phase oscillations of the voltage signal are observed [compare Figs. 4(b) and 4(c)].

Next, we consider the case where generation of $\mathrm{V}-\mathrm{Av}$ pairs takes place even without varying the boundary conditions (which goes beyond the DCE analogy, though it can be considered as a classical analog of a "superradiance vacuum state" [20]). Figure 5(a) shows the $n(v)$ curve of the system for the applied current $j=1.01 j_{c}$ and for the same values of the magnetic field as in Fig. 4. For this value of the current we have preformed $\mathrm{V}$-Av pairs, and in the presence of the ac 

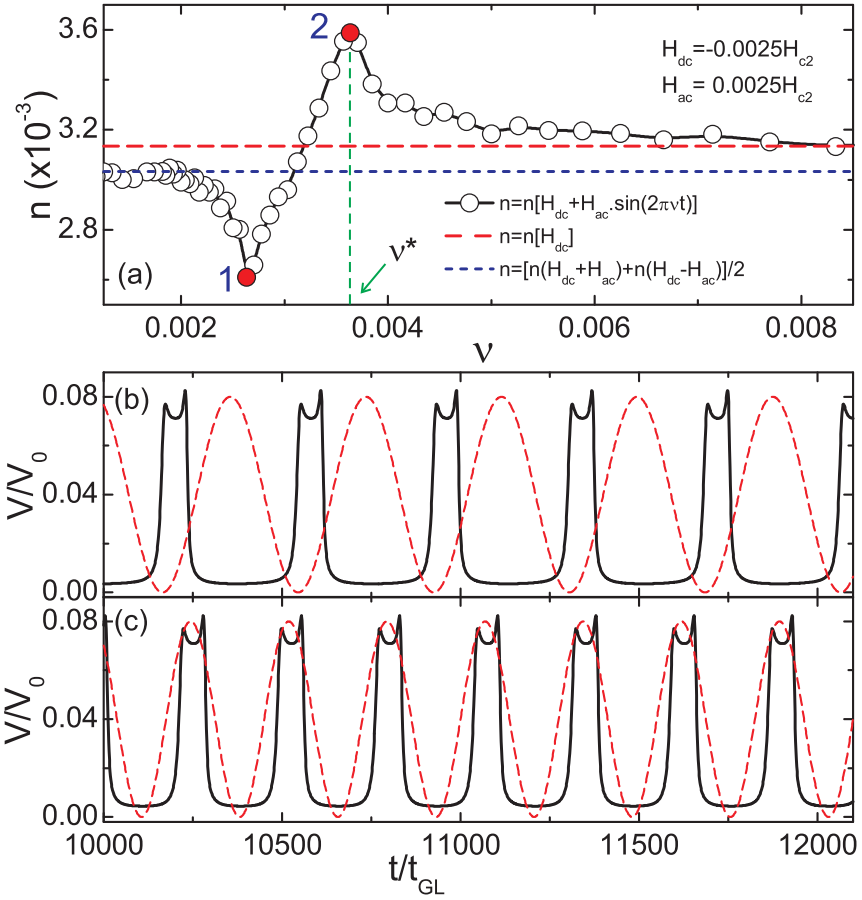

FIG. 5. (Color online) (a) $n(v)$ characteristics of the sample at $j=1.01 j_{c}$ for $H_{\mathrm{dc}}=-0.0025 H_{c 2}$ and $H_{\mathrm{ac}}=0.0025 H_{c 2}$ (cf. Fig. 4). (b, c) $V(t)$ characteristics of the sample for the frequency of the magnetic field indicated in panel (a) by points 1 and 2, respectively. Dashed/red curves show the profile of the ac magnetic field in arbitrary units.

magnetic field we find qualitatively different results: (i) a dip in the $n(v)$ curve is observed which is followed by a clear maximum in the pair creation rate arising with increasing field frequency, and (ii) two different plateaus are observed in the field dependence of the pair generation away from the resonance area. Both at the minimum and maximum of the $n(v)$ curve we notice clear phase-locked dynamics of fluxons, which is evident from the voltage vs time characteristics of the system [see Figs. 5(b) and 5(c)]. The value of $n_{\max }$ is obtained at the resonance frequency $v^{*}=v_{0} / 2$, where $v_{0}$ is the characteristic frequency of the system at $H=H_{\mathrm{dc}}$ determined by the crossing of the Josephson fluxons across the system [i.e., $v_{0}=1 / \delta t$, see Fig. 3(a)]. Frequency $v^{*}$ increases slightly with increasing $H_{\mathrm{dc}}$ [see Fig. 3(b)]. At larger frequencies, $n(v)$ equals the number of fluxons generated in a dc magnetic field $n=n\left(H_{\mathrm{dc}}\right)$ (see horizontal dashed/red line), whereas $n(v)$ approaches the value obtained in the adiabatic regime $n=\left[n\left(H_{\mathrm{dc}}+H_{\mathrm{ac}}\right)+n\left(H_{\mathrm{dc}}-H_{\mathrm{ac}}\right)\right] / 2$ (see horizontal dotted/blue line) at smaller frequencies of the applied magnetic field. We find about a $20 \%$ increase of the number of fluxons created during the parametric conversion process as compared to the adiabatic case.

To see the effect of the frequency of the ac magnetic field $v$ on the spectrum of the flux-flow induced radiation, we have calculated the Fourier power spectrum of the voltage signal, which is shown in Fig. 6 for four values of $v$. At low frequencies of the magnetic field the spectrum is characterized by two pronounced peaks at $v=0.00305 / t_{\mathrm{GL}}$ and $v=0.0043 / t_{\mathrm{GL}}$ with additional (mostly broad and irregular) small peaks at

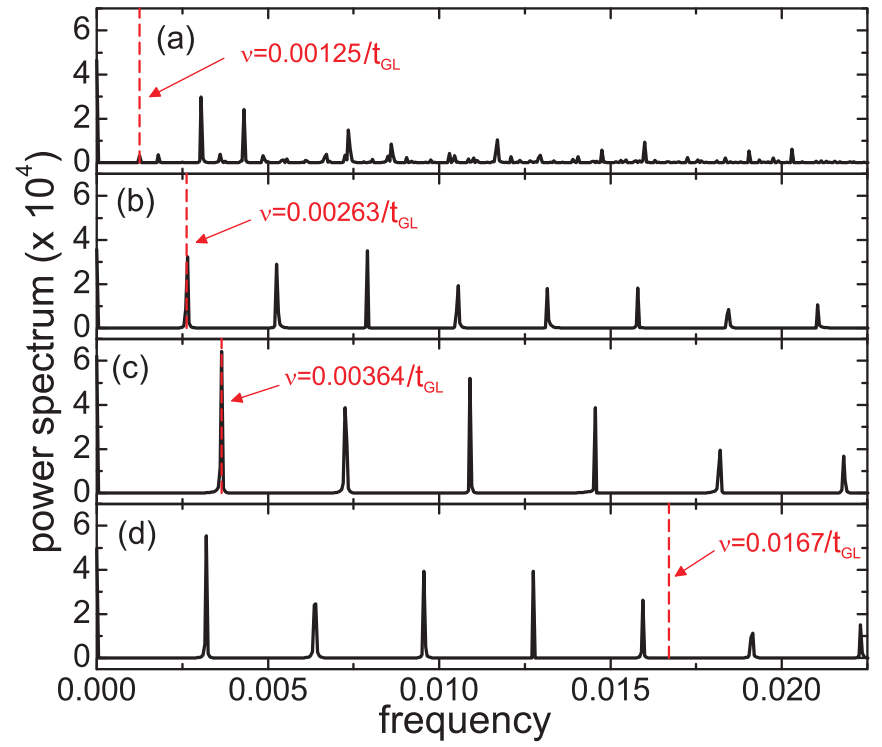

FIG. 6. (Color online) Fourier power spectrum of the voltage for four different values of the frequency of the ac magnetic field $H / H_{c 2}=-0.0025+0.0025 \sin (2 \pi v t)$, as indicated by vertical dashed lines.

larger frequencies [Fig. 6(a)]. With increasing $v$ the first peak appears at resonance frequency [vertical dashed curve in Fig. 6(b)]. The other peaks are all equidistant and well separated. The largest intensity of the spectral lines is observed for the characteristic frequency $v^{*}=0.00364 / t_{\mathrm{GL}}$ [Fig. 6(c)], which is also the frequency for the largest nucleation rate of the V-Av pairs [see Fig. 5(a)]. The distance between the spectral lines is also maximal for this frequency. The intensity of the lines decreases again and they are detected at smaller frequencies with further increasing $v$ [Fig. 6(d)]. No peak in the spectrum is found corresponding to the frequency of the external drive.

\section{CONCLUSIONS}

In summary, we have shown how the Josephson V-Av pairs are generated in layered superconducting systems by applied electric current and how their nucleation and dynamics are affected by dc out-of-plane magnetic field and pinned Abrikosov vortices. More importantly, we have shown that nucleation of the pairs can be triggered and broadly controlled by the ac magnetic field, which is in fact a neat realization of parametric amplification, resembling the phenomena seen in the dynamic Casimir effect in optical systems. Here the ac magnetic field provides a time-dependent boundary condition for the generation of $\mathrm{V}-\mathrm{Av}$ pairs, which mimic in behavior the photons created in vibrating optical cavities. We predicted a clear resonance effect between the frequency of generated pairs and the frequency of the ac field. Even though the parametric amplification of generated $\mathrm{V}$-Av pairs is essentially a classical effect, which somewhat restricts a proposed analogy with the dynamic Casimir effect and its known quantum analogs [18-24], this could be considered as an advantage since the proposed phenomenon can be more easily realized in experiment and still elucidate several intriguing properties 
of the DCE. We also reported unusual properties which are reminiscent of the "superradiant" state, when pairs exist in the system due to applied current even before applying the ac field. Finally we showed how the spectrum of flux-flow induced radiation is affected by the frequency of the ac field. These findings may have important implications for the design of tunable high-frequency electromagnetic radiation sources. Our analysis revealed controllability of the frequency of Josephson oscillations with over $80 \%$ by changing just the magnetic field, whereas the maximum $40 \%$ tunability has been achieved to date (by changing bias current and bath temperature, see Ref. [56]).

\section{ACKNOWLEDGMENTS}

This work was supported by the Flemish Science Foundation (FWO-Vl) and the Leverhulme Trust. G.R.B. acknowledges support from a EU-Marie Curie individual grant (Grant No. 253057)
[1] B. R. Mollow and R. J. Glauber, Phys. Rev. 160, 1076 (1967), references therein.

[2] M. A. Castellanos-Beltran, K. D. Irwin, G. C. Hilton, L. R. Vale, and K. W. Lehnert, Nat. Phys. 4, 929 (2008).

[3] B. H. Eom, P. K. Day, H. G. LeDuc, and J. Zmuidzinas, Nat. Phys. 8, 623 (2012).

[4] T. Yamamoto, K. Inomata, M. Watanabe, K. Matsuba, T. Miyazaki, W. D. Oliver, Y. Nakamura, and J. S. Tsai, Appl. Phys. Lett. 93, 042510 (2008); M. A. Castellanos-Beltran and K. W. Lehnert, ibid. 91, 083509 (2007).

[5] R. L. Byer, in Quantum Electronics, edited by H. Rabin and C. L. Tang (Academic Press, New York, 1975), Vol. 1.

[6] B. D. Gomperts, I. M. Kramer, and P. E. R. Tatham, Signal Transduction (Academic Press, London, 2002).

[7] S. Savel'ev, A. L. Rakhmanov, and F. Nori, Phys. Rev. E 72, 056136 (2005); S. Savel'ev, A. M. Zagoskin, A. L. Rakhmanov, A. N. Omelyanchouk, Z. Washington, and F. Nori, Phys. Rev. A 85, 013811 (2012).

[8] G. T. Moore, J. Math. Phys. (N. Y.) 11, 2679 (1970).

[9] Yu. E. Lozovik, V. G. Tsvetus, and E. A. Vinogradov, JETP Lett. 61, 723 (1995).

[10] E. Yablonovitch, Phys. Rev. Lett. 62, 1742 (1989).

[11] F. X. Dezael and A. Lambrecht, Europhys. Lett. 89, 14001 (2010).

[12] V. V. Dodonov, Phys. Scr. 82, 038105 (2010).

[13] P. D. Nation, J. R. Johansson, M. P. Blencowe, and F. Nori, Rev. Mod. Phys. 84, 1 (2012).

[14] C. Braggio, G. Bressi, G. Carugno, A. Lombardi, A. Palmieri, G. Ruoso, and D. Zanello, Rev. Sci. Instrum. 75, 4967 (2004); Europhys. Lett. 70, 754 (2005).

[15] C. M. Wilson, G. Johansson, A. Pourkabirian, M. Simoen, J. R. Johansson, T. Duty, F. Nori, and P. Delsing, Nature (London) 479, 376 (2011).

[16] J.-C. Jaskula, G. B. Partridge, M. Bonneau, R. Lopes, J. Ruaudel, D. Boiron, and C. I. Westbrook, Phys. Rev. Lett. 109, 220401 (2012).

[17] I. Carusotto, R. Balbinot, A. Fabbri, and A. Recati, Eur. Phys. J. D 56, 391 (2009).

[18] J. R. Johansson, G. Johansson, C. M. Wilson, and F. Nori, Phys. Rev. Lett. 103, 147003 (2009).

[19] S. De Liberato, D. Gerace, I. Carusotto, and C. Ciuti, Phys. Rev. A 80, 053810 (2009).

[20] G. Vacanti, S. Pugnetti, N. Didier, M. Paternostro, G. M. Palma, R. Fazio, and V. Vedral, Phys. Rev. Lett. 108, 093603 (2012).

[21] A. V. Dodonov and V. V. Dodonov, Phys. Rev. A 85, 063804 (2012).
[22] L. J. Garay, J. R. Anglin, J. I. Cirac, and P. Zoller, Phys. Rev. Lett. 85, 4643 (2000).

[23] R. Balbinot, A. Fabbri, S. Fagnocchi, A. Recati, and I. Carusotto, Phys. Rev. A 78, 021603 (2008).

[24] O. Lahav, A. Itah, A. Blumkin, C. Gordon, S. Rinott, A. Zayats, and J. Steinhauer, Phys. Rev. Lett. 105, 240401 (2010).

[25] M. V. Milošević and F. M. Peeters, Phys. Rev. B 68, 024509 (2003); Phys. Rev. Lett. 93, 267006 (2004); 94, 227001 (2005); M. V. Milošević, G. R. Berdiyorov, and F. M. Peeters, Phys. Rev. B 75, 052502 (2007); M. V. Milošević, F. M. Peeters, and B. Jankó, Supercond. Sci. Technol. 24, 024001 (2011).

[26] J. S. Neal, M. V. Milošević, S. J. Bending, A. Potenza, L. San Emeterio, and C. H. Marrows, Phys. Rev. Lett. 99, 127001 (2007); R. B. G. Kramer, A. V. Silhanek, W. Gillijns, and V. V. Moshchalkov, Phys. Rev. X 1, 021004 (2011); M. Iavarone, A. Scarfato, F. Bobba, M. Longobardi, G. Karapetrov, V. Novosad, V. Yefremenko, F. Giubileo, and A. M. Cucolo, Phys. Rev. B 84, 024506 (2011).

[27] M. V. Milošević, G. R. Berdiyorov, and F. M. Peeters, Phys. Rev. Lett. 95, 147004 (2005); M. V. Milošević and F. M. Peeters, Physica C 437, 208 (2006); C. L. S. Lima and C. C. de Souza Silva, Phys. Rev. B 80, 054514 (2009); V. N. Gladilin, J. Tempere, J. T. Devreese, W. Gillijns, and V. V. Moshchalkov, ibid. 80, 054503 (2009); A. V. Silhanek, V. N. Gladilin, J. Van de Vondel, B. Raes, G. W. Ataklti, W. Gillijns, J. Tempere, J. T. Devreese, and V. V. Moshchalkov, Supercond. Sci. Technol. 24, 024007 (2011); A. V. Kapra, V. R. Misko, D. Y. Vodolazov, and F. M. Peeters, ibid. 24, 024014 (2011); B. R. C. H. T. de Aquino, L. R. E. Cabral, C. C. de Souza Silva, J. Albino Aguiar, M. V. Milošević, and F. M. Peeters, Physica C 479, 115 (2012).

[28] See, e.g., T. Winiecki, B. Jackson, J. F. McCann, and C. S. Adams, J. Phys. B 33, 4069 (2000).

[29] G. R. Berdiyorov, M. V. Milošević, and F. M. Peeters, Phys. Rev. B 79, 184506 (2009).

[30] G. R. Berdiyorov, M. V. Milošević, L. Covaci, and F. M. Peeters, Phys. Rev. Lett. 107, 177008 (2011).

[31] M. V. Fistul, A. Wallraff, Y. Koval, A. Lukashenko, B. A. Malomed, and A. V. Ustinov, Phys. Rev. Lett. 91, 257004 (2003).

[32] S. Savel'ev, V. A. Yampol'skii, A. L. Rakhmanov, and F. Nori, Rep. Prog. Phys. 73, 026501 (2010); M. Tachiki, M. Iizuka, K. Minami, S. Tejima, and H. Nakamura, Phys. Rev. B 71, 134515 (2005); S. Savel'ev, V. Yampol'skii, A. Rakhmanov, and F. Nori, ibid. 72, 144515 (2005); A. L. Rakhmanov, S. E. 
Savel'ev, and F. Nori, ibid. 79, 184504 (2009); F. Marchesoni, S. Savel'ev, M. Tachiki, and F. Nori, ibid. 81, 174531 (2010); S. Savel'ev, A. L. Rakhmanov, and F. Nori, Phys. Rev. Lett. 94, 157004 (2005); Phys. Rev. B 74, 184512 (2006).

[33] S. A. Fulling and P. C. W. Davies, Proc. R. Soc. London, Ser. A 348, 393 (1976).

[34] A. Lambrecht, M. T. Jaekel, and S. Reynaud, Phys. Rev. Lett. 77, 615 (1996).

[35] V. M. Krasnov, Phys. Rev. B 83, 174517 (2011); 85, 134525 (2012).

[36] T. Kashiwagi, K. Yamaki, M. Tsujimoto, K. Deguchi, N. Orita, T. Koike, R. Nakayama, H. Minami, T. Yamamoto, R. A. Klemm, M. Tachiki, and K. Kadowaki, J. Phys. Soc. Jpn. 80, 094709 (2011).

[37] M. Li, J. Yuan, N. Kinev, J. Li, B. Gross, S. Guenon, A. Ishii, K. Hirata, T. Hatano, D. Koelle, R. Kleiner, V. P. Koshelets, H. Wang, and P. Wu, Phys. Rev. B 86, 060505(R) (2012).

[38] M.-H. Bae, H.-J. Lee, and J.-H. Choi, Phys. Rev. Lett. 98, 027002 (2007).

[39] M. Tsujimoto, T. Yamamoto, K. Delfanazari, R. Nakayama, T. Kitamura, M. Sawamura, T. Kashiwagi, H. Minami, M. Tachiki, K. Kadowaki, and R. A. Klemm, Phys. Rev. Lett. 108, 107006 (2012).

[40] G. R. Berdiyorov, S. E. Savel'ev, M. V. Milošević, F. V. Kusmartsev, and F. M. Peeters, Phys. Rev. B 87, 184510 (2013).

[41] L. Kramer and R. J. Watts-Tobin, Phys. Rev. Lett. 40, 1041 (1978); R. J. Watts-Tobin, Y. Krahenbuhl, and L. Kramer, J. Low Temp. Phys. 42, 459 (1981).

[42] A. I. Gubin, K. S. Il'in, S. A. Vitusevich, M. Siegel, and N. Klein, Phys. Rev. B 72, 064503 (2005).

[43] A. D. Hernández, B. J. Baelus, D. Domínguez, and F. M. Peeters, Phys. Rev. B 71, 214524 (2005).
[44] T. Golod, A. Rydh, and V. M. Krasnov, Phys. Rev. Lett. 104, 227003 (2010).

[45] A. V. Ustinov, T. Doderer, B. Mayer, R. P. Huebener, A. A. Golubov, and V. A. Oboznov, Phys. Rev. B 47, 944 (1993).

[46] A. E. Koshelev, Phys. Rev. Lett. 83, 187 (1999); Phys. Rev. B 68, 094520 (2003); J. Mirković, S. E. Savel'ev, E. Sugahara, and K. Kadowaki, Phys. Rev. Lett. 86, 886 (2001); S. E. Savel'ev, J. Mirković, and K. Kadowaki, Phys. Rev. B 64, 094521 (2001); S. Savel'ev and F. Nori, Nat. Mater. 1, 179 (2002); D. Cole, S. Bending, S. Savel'ev, A. Grigorenko, T. Tamegai, and F. Nori, ibid. 5, 305 (2006).

[47] A. E. Koshelev, Yu. I. Latyshev, and M. Konczykowski, Phys. Rev. B 74, 104509 (2006).

[48] A. E. Koshelev, A. I. Buzdin, I. Kakeya, T. Yamamoto, and K. Kadowaki, Phys. Rev. B 83, 224515 (2011).

[49] A. V. Samokhvalov, A. S. Mel'nikov, and A. I. Buzdin, Phys. Rev. B 85, 184509 (2012).

[50] N. Uchida, K. Enpuku, Y. Matsugaki, S. Tomita, and F. Irie, J. Appl. Phys. 54, 5287 (1983).

[51] S. L. Miller, Kevin R. Biagi, John R. Clem, and D. K. Finnemore, Phys. Rev. B 31, 2684 (1985).

[52] A. A. Golubov and M. Yu. Kupriyanov, Zh. Eksp. Teor. Fiz. 92, 1512 (1987); J. Low Temp. Phys. 70, 83 (1988).

[53] L. G. Aslamazov and E. V. Gurovich, Pis'ma Zh. Eksp. Teor. Fiz. 40, 22 (1984) [Sov. Phys. JETP Lett. 40, 746 (1984)].

[54] B. Jackson, J. F. McCann, and C. S. Adams, Phys. Rev. Lett. 80, 3903 (1998).

[55] V. V. Dodonov, Phys. Lett. A 207, 126 (1995); V. V. Dodonov and A. B. Klimov, Phys. Rev. A 53, 2664 (1996).

[56] H. B. Wang, S. Guénon, B. Gross, J. Yuan, Z. G. Jiang, Y. Y. Zhong, M. Grünzweig, A. Iishi, P. H. Wu, T. Hatano, D. Koelle, and R. Kleiner, Phys. Rev. Lett. 105, 057002 (2010). 